\title{
Modeling of equivalent ionospheric currents from meridian magnetometer chain data
}

\author{
V. A. Popov*, V. O. Papitashvili**, and J. F. Watermann \\ Danish Meteorological Institute, Lyngbyvej 100, Copenhagen, DK-2100, Denmark
}

(Received May 26, 2000; Revised September 6, 2000; Accepted September 19, 2000)

\begin{abstract}
In recent years, quantitative analysis of the magnetosphere-ionosphere coupling and electrodynamics of the polar ionosphere received much attention. Though remarkable progress has been made in this field by using a variety of magnetogram inversion techniques in order to infer the global ionospheric current distribution, there is still a need for modeling ionospheric currents locally, over a certain region, for comparison with other geophysical ground-based and satellite observations. This paper presents a simple method for estimating equivalent ionospheric currents using magnetic field observations along a meridian chain of ground-based vector magnetometers. The method can be applied in an automatic fashion to any available magnetometer chain data, for example, from the DMI Greenland west coast chain. We first describe how we separate contributions to the observed geomagnetic variations from external (ionospheric) and internal (induced) sources. We then model the ionospheric electrojet by a sequence of narrow current strips and apply the Biot-Savart law to formulate an inversion problem. Using a regularization technique, we find a stable distribution of the equivalent ionospheric currents crossing the magnetometer chain in eastward and westward direction. Simulation tests and a case study (20 March 1999) are discussed in order to illustrate properties of the solution to the inverse problem and to present a practical tool, which is accessible through the DMI World Wide Web site.
\end{abstract}

\section{Introduction}

Investigations of intensity and spatial distribution of ionospheric Hall currents over the polar regions (known as "polar" or "auroral" electrojets) are important for better understanding of the magnetosphere-ionosphere coupling. Various methods of the current reconstruction from ground magnetometer data have extensively been discussed in the literature. In early papers, the polar electrojet was modeled by single or double current strips (e.g., Pudovkin, 1960; Kisabeth and Rostoker, 1971; Korobeinikov et al., 1977; Kuznetsov et al., 1978). Mersmann et al. (1979) performed upward continuation of the observed magnetic field to estimate the current distribution. Application of that technique builds on the assumption that the ionospheric sheet current does not vary in the direction perpendicular to the magnetometer chain. Subsequently, different techniques (some of which make assumptions about ionospheric conductivities) were used to calculate currents over the entire polar region (e.g., Kamide et al., 1981) and on a regional scale (e.g., Baumjohann et al., 1981). Richmond and Baumjohann (1983) and Walker et al. (1997) discussed application of the spherical cap harmonic analysis; a powerful analytical method to reconstruct ionospheric currents from spatially distributed data. An overview over various techniques used in the past was given by Untiedt and Baumjohann (1993). Recently, a different approach to

\footnotetext{
*Also at IZMIRAN, Troitsk, Moscow Region, Russia.

**Also at SPRL, University of Michigan, Ann Arbor, Michigan, U.S.A.
}

Copy right $($ C) The Society of Geomagnetism and Earth, Planetary and Space Sciences (SGEPSS); The Seismological Society of Japan; The Volcanological Society of Japan; The Geodetic Society of Japan; The Japanese Society for Planetary Sciences. the field continuation was taken by Amm (1997) and Amm and Viljanen (1999); they construct elementary ionospheric current systems, the divergence-free part of which is associated with a ground magnetic field, and derive their distribution in the ionosphere. Boteler and Pirjola (1998) and Viljanen et al. (1999) studied various models of ionospheric currents in order to investigate the geomagnetically induced ground currents.

Following the approach suggested for the current reconstruction by the work from the 1970s, Kotikov et al. (1987, 1991) developed a simple but practical inverse scheme to infer the auroral electrojet fine structure by utilizing a series of linear ionospheric currents with different intensities, evenly distributed over a certain distance at 100-km altitude. The current distribution was adjusted such that it would fit measurements made at the Earth's surface. This method defines currents at an arbitrary number of points (50 in their case) using only a few stations located along the geomagnetic meridian. Olsen (1996) used a similar approach to determine ionospheric currents from satellite magnetic field observations. Popov and Feldstein (1996) suggested a refinement of Kotikov's method by approximating the auroral electrojets with a series of narrow current strips of a finite width but of different intensities, distributed "side-by-side" along a geomagnetic meridian at $115-\mathrm{km}$ altitude over the range of latitudes covered by the ground stations.

The goal of our paper is two-fold. First, we apply the above-mentioned refined method to a number of sets of simulated data in order to study the method's accuracy, spatial resolution, and properties in detail, having in mind the de- 
velopment of a practical, useful algorithm for automatic estimation of equivalent ionospheric currents from any meridian chain data. Then we apply the technique to a data set from 20 March 1999, obtained from the Greenland west coast magnetometer chain, in order to infer the polar electrojet pattern and to study how the pattern evolves over the course of the day. The example shows the usefulness of the method in applying it to the Greenland west coast magnetometers through the Danish Meteorological Institute (DMI) World Wide Web site, http: //www. dmi.dk/projects/chain/.

\section{Modeling Method and Inversion Technique 2.1 Initial assumptions}

The method described in this study was developed for computing the distribution of the equivalent ionospheric current crossing a geomagnetic meridian. In this method, we use the following a priori assumptions:

- The equivalent ionospheric currents are sheet currents flowing at a fixed altitude of $115 \mathrm{~km}$ above a planar ground and perpendicular to the meridian chain of magnetometers.

- The currents are composed of a spatial sequence of 100 narrow, infinitely long strips; each strip carries a current of a certain density that is either eastward or westward oriented and change with time.

- The observed ground magnetic fields are produced solely by these ionospheric currents and their associated ground-induced currents; there are no other sources for magnetic field variations.

- The electrical conductivity beneath the Earth's surface does not vary in the direction perpendicular to the magnetometer chain.

The result obtained is a set of 100 time-dependent strip current intensities.

The Greenland west coast magnetometer chain was selected for the analysis since it extends over a wide range of corrected geomagnetic (CGM) latitudes (Gustafsson et al., 1992). The chain consists of 12 stations located along $\sim 40^{\circ}$ CGM longitude and almost evenly distributed between $66.3^{\circ}$ (Narsarsuaq, NAQ) and 85.4 (Qaanaaq, THL) CGM latitude. This distribution of stations provides an excellent opportunity to model equivalent ionospheric currents from auroral latitudes deep into the polar cap. In the following subsections, we describe the algorithm in detail.

\subsection{Separation of external and internal fields}

We suppose that the ionospheric currents cause variations in the vertical ( $\mathrm{Z}$-downward) and horizontal ( $H$-northward along the mean local magnetic meridian, $E$-eastward) magnetic field components recorded at the ground observation points. The ionospheric current pattern that is consistent with the above-mentioned assumptions does not generate a magnetic field component aligned with the current strips, i.e., perpendicular to the meridian chain. We limit our considerations to the magnetic field fluctuations with periods exceeding some $30 \mathrm{~min}$ in order to ensure that the induced currents flow at depths much deeper than that of the ocean (e.g., Engels, 1997). In that case, the land/sea conductivity discontinuity at the Greenland west coast has little effect on the ground magnetic variations.

The observed magnetic field at each station and each time sample can be written as:

$$
\begin{aligned}
& H=H_{\text {ext }}+H_{i n t} \\
& Z=Z_{\text {ext }}+Z_{\text {int }}
\end{aligned}
$$

where indices ext and int are assigned to the geomagnetic variations from the external and internal sources, respectively. (Since this applies to any arbitrary point in time, $t$, we suppress the time parameter throughout this paper.) According to Pudovkin (1960), Weaver (1964), and Mersmann et al. (1979), the differences between magnetic fields caused by external and induced sources read at any point, $l$, along the meridian:

$$
\begin{aligned}
& H_{\text {ext }}(l)-H_{\text {int }}(l)=\frac{1}{\pi} \int_{-\infty}^{\infty} \frac{Z(\xi)}{\xi-l} d \xi \\
& Z_{\text {int }}(l)-Z_{\text {ext }}(l)=\frac{1}{\pi} \int_{-\infty}^{\infty} \frac{H(\xi)}{\xi-l} d \xi
\end{aligned}
$$

Here $H$ and $Z$ represent the cumulative magnetic effect from all ionospheric current strips and their induced counterparts at any given point, $l$, along the meridian. Combining (1) and (2), we may calculate the external contribution to the corresponding geomagnetic field components as:

$$
\begin{aligned}
& H_{\text {ext }}(l)=\frac{1}{2}\left[H(l)+\frac{1}{\pi} \int_{-\infty}^{\infty} \frac{Z(\xi)}{\xi-l} d \xi\right] \\
& Z_{\text {ext }}(l)=\frac{1}{2}\left[Z(l)-\frac{1}{\pi} \int_{-\infty}^{\infty} \frac{H(\xi)}{\xi-l} d \xi\right]
\end{aligned}
$$

For the numerical computation of the integrals, we construct a continuous distribution of the magnetic field variation along the meridian by fitting a cubic spline to the measurements at the spaced sites. The choice of a specific spline function is not critical; we tested a few different standard and specifically-constructed spline functions, but found that differences in numerical integration are less than $0.5 \%$. The representation of $H$ and $Z$ through continuous piecewise cubic polynomials (splines) guarantees that the integrals converge if the singularity at $\xi=l$ is evaluated using Cauchy's principal value. The entire latitude range covered here is divided into 1201 evenly spaced integration nodes which are about $2.5 \mathrm{~km}$ apart. In order to apply Cauchy's principal value theorem the integration nodes must lie symmetrically on both sides of the reference point, $l$. When performing the numerical integration we omit the two segments adjacent to the reference point, i.e., within $l \pm 2.5 \mathrm{~km}$.

The integrals in Eq. (3) are computed over the latitudes from $62^{\circ}$ to $90^{\circ}$ (i.e., $\pm 4^{\circ}$ beyond the range occupied by the magnetometer sites); here we assume that the magnitude of magnetic field variations outside of this (extended) region is negligible. In order to achieve smoothness of the field beyond the equatorward and poleward boundaries of the 
magnetometer chain, we set the magnitude of $H_{+4}$ (at the end points of our simulation interval) to be $10 \%$ of the $H$ values at the highest and lowest stations, respectively. The magnitude $Z_{ \pm 4}$ is set to be $40 \%$ of the $Z$ values of the highest and lowest stations. As a result, the reconstructed $H$ component is slightly smaller in magnitude (by up to $8 \%$ ) than the true $H$ component, and the reconstructed $Z$ component is slightly larger than the true $Z$ component by about the same amount. This applies to the entire latitude range covered by the meridian chain. The additional nodes, $H_{ \pm 4^{\circ}}$ and $Z_{ \pm 4^{\circ}}$, are used only for the field separation, i.e., Eq. (3). The inversion scheme described below uses only data from the magnetometer stations and not from the extrapolated end points.

\subsection{Modeling of the equivalent ionospheric current dis- tribution}

For modeling the equivalent ionospheric current, we distribute 100 east-west-oriented current strips evenly along the meridian confined between $2^{\circ}$ equatorward of the lowest and $2^{\circ}$ poleward of the highest latitude stations; that kind of extension is needed to reproduce the currents properly at the edges of the chain. The current density and orientation may change from one strip to another, but they are uniform within each strip. According to the Biot-Savart law, we can calculate magnetic field disturbances at any point $l$ on the ground along the geomagnetic meridian from every single current strip:

$$
\begin{aligned}
& H_{\text {ext }}(l)=\frac{j_{i}}{2 \pi}\left(\arctan \frac{x_{i}+d}{h}-\arctan \frac{x_{i}-d}{h}\right) \\
& Z_{\text {ext }}(l)=\frac{j_{i}}{4 \pi} \ln \left(\frac{h^{2}+\left(x_{i}+d\right)^{2}}{h^{2}+\left(x_{i}-d\right)^{2}}\right)
\end{aligned}
$$

where $j_{i}$ is the current density in the $i^{t h}$ strip, $d$ the halfwidth of the strip, $h$ its altitude, and $x_{i}$ is the distance from the observation point to the ground projection of the center of the $i^{\text {th }}$ strip.

Let $K$ be the number of magnetometers in the chain. At any observation point, $k(k=1, \ldots, K)$, the magnetometer senses the magnetic field from all current strips:

$$
\begin{aligned}
H_{k}^{e x t}= & \frac{1}{2 \pi} \sum_{i=1}^{N} j_{i}\left(\arctan \frac{x_{i k}+d}{h}\right. \\
& \left.-\arctan \frac{x_{i k}-d}{h}\right) \\
Z_{k}^{\text {ext }}= & \frac{1}{4 \pi} \sum_{i=1}^{N} j_{i} \ln \left(\frac{h^{2}+\left(x_{i k}+d\right)^{2}}{h^{2}+\left(x_{i k}-d\right)^{2}}\right)
\end{aligned}
$$

Here $N=100$ is the horizontal distance from the $k^{\text {th }}$ observation point to the center of the $i^{\text {th }}$ current strip, $H_{k}$ and $Z_{k}$ are the horizontal and vertical magnetic field components, respectively, and $j_{i}$ is the current density in the $i^{\text {th }}$ strip. This yields $2 K$ equations to find $N$ current densities. When $2 K$ is less than $N$, the problem is underdetermined, and the solution space is manifold. In order to constrain the solution space we use the regularization method developed by Tikhonov and Arsenin (1977).

For this purpose, we construct residual functions, $Q_{H}$ and $Q_{Z}$, which provide a measure of the difference between ob- served and modeled ground magnetic fields plus a "regularization" term; these functions must be non-negative:

$$
\begin{aligned}
Q_{H}= & \sum_{k=1}^{L}\left(H_{k}^{e x t}-\frac{1}{2 \pi} \sum_{i=1}^{N} j_{i}\left(\arctan \frac{x_{i k}+d}{h}\right.\right. \\
& \left.\left.-\arctan \frac{x_{i k}-d}{h}\right)\right)^{2}+\alpha \sum_{i=1}^{N-1}\left(j_{i+1}-j_{i}\right)^{2} \\
Q_{Z}= & \sum_{k=1}^{L}\left(Z_{k}^{e x t}-\frac{1}{4 \pi} \sum_{i=1}^{N} j_{i} \ln \left(\frac{h^{2}+\left(x_{i k}+d\right)^{2}}{h^{2}+\left(x_{i k}-d\right)^{2}}\right)\right)^{2} \\
& +\beta \sum_{i=1}^{N} j_{i}^{2}
\end{aligned}
$$

By minimizing any of the two functions, $Q_{H}$ or $Q_{Z}$, we seek current distributions which return magnetic field values close to those observed at the $K$ sites, and in which either the cumulative squared differences between current intensities in adjacent strips are minimal (6), or the total squared current over all strips is minimal (7). The choice of the "regularization parameters", $\alpha$ and $\beta$, reflects a trade-off between solutions matching more accurately the observed magnetic field and those resulting in smoother current distributions.

Note that this procedure can be applied separately to either one of the observed $H$ and $Z$ magnetic field components. If contributions from magnetic field sources, which may not fulfill the model assumptions, are negligible, the $H$-based and $Z$-based reconstructions are almost identical. A significant mismatch between the currents reconstructed from the $H$ and $Z$ components may indicate violation of our initial assumptions, e.g., contributions from currents flowing along or parallel to the magnetometer chain, and/or from currents flowing beyond the poleward and equatorward boundaries of the chain. Therefore, we recommend to utilize both equations (6) and (7) independently for the electrojet reconstruction and then compare the results, visually or numerically, in order to assess the level of confidence for the obtained current density profiles.

The first derivatives with respect to $j_{i}$ of either one of equations (6) and (7), when equated to zero, form a set of 100 (constrained) normal equations of the problem. From this set of normal equations we may determine the minima of the functions $Q_{H}$ and $Q_{Z}$. According to Tikhonov and Arsenin (1977), the parameters $\alpha$ and $\beta$ can be chosen such that the discrepancy between observed and modeled magnetic field values takes a minimum. In our case we found that $\alpha$ and $\beta$ are to be in the range, $10^{-6}$ to $10^{-9}$. We choose $\alpha=\beta=10^{-6}$ for all of the following tests we performed in this study. Because proper selection of these parameters depends on the geometric distribution of observational points, the parameters should be chosen once for a given distribution of the stations.

\section{Results from Simulation Tests}

In our simulation, we used a current flowing perpendicular to the chain, with the density distribution along the meridian given by:

$$
j(l)=j_{0} e^{-\frac{\left(l_{0}-l\right)^{2}}{d_{0}^{2}}}
$$


where $l_{0}$ fixes the current's center, $2 \cdot d_{0}$ is its effective width, and the current is placed at an altitude of $h=115 \mathrm{~km}$ above the planar ground. The induced currents are assumed to flow in a perfectly conducting layer at a depth of $300 \mathrm{~km}$ (e.g., Mareschal, 1976; Richmond and Baumjohann, 1983). For the simulation, we used twelve "virtual magnetometers" distributed evenly between $66^{\circ}$ and $86^{\circ}$ CGM latitude. The latitudinal spacing between these "stations" is $1.82^{\circ}$ (where $1^{\circ} \approx 111 \mathrm{~km}$ ); that distance approximates the actual distribution of DMI's magnetometers along the Greenland west coast.

Figure 1 shows current distributions modeled using Eq. (6) but with different values for the regularization parameter $\alpha\left(\alpha=0,10^{-6}\right.$, and 1). A simulation current of the type Eq. (8) was used, with $j_{0}=1 \mathrm{~A} / \mathrm{m}$ and $d_{0}=1.8^{\circ}$. First, the ground magnetic field at the $K$ virtual magnetometer sites is computed. Then the induced and ionospheric parts are separated using Eq. (3), and finally the current distribution is reconstructed using the regularization scheme, i.e. Eq. (6). The top panel shows one of many possible reconstructed current distributions for $\alpha=0$. Its magnetic field at all 12 virtual magnetometer sites is identical to that from the original current. However, it is obvious that the reconstructed current has little in common with the bell-shaped original distribution. The middle and bottom panels show that the reconstructed distribution becomes smoother with increasing $\alpha$.

We tested the algorithm's capability to resolve a double- peak initial current distribution by simulating a sheet current composed of two Gaussian distributions (cf., Eq. (8)) with equal amplitudes, $j_{0}=1 \mathrm{~A} / \mathrm{m}$ and equal widths, $d_{0}=1.8^{\circ}$. Their centers were separated by $1.5,1.75$, and 2.0 times $1.82^{\circ}$ (the distance between ground "stations"). The original current distribution is practically flat between the peaks in the first case $\left(1.5 \cdot 1.82^{\circ}\right.$ spacing $)$, forms a shallow valley between the two peaks in the second case $\left(1.75 \cdot 1.82^{\circ}\right.$ spacing $)$, and exhibits a deeper valley in the third case $\left(2.0 \cdot 1.82^{\circ}\right.$ spacing $)$, see Fig. 2. These different distributions were simulated such that one of the two peaks remains located directly above one of the innermost ground stations and the second one is stepwise shifted away from the first one.

Reconstructed current distributions were obtained using the northward magnetic field component, cf., Eq. (6), and the vertical component, cf., Eq. (7), independently. Figure 2 shows the original and reconstructed distributions when the peak spacing is $1.5 \cdot 1.82^{\circ}$ (top panel), $1.75 \cdot 1.82^{\circ}$ (center), and $2.0 \cdot 1.82^{\circ}$ (bottom panel). If the distance between two peaks is large enough so that they can be distinguished in the original current distribution, the reconstruction algorithm (using the $H$ component) is also capable to resolve the peaks. Note that the algorithm's capability to resolve fine structures such as double peaks depends not only on the spacing between ground stations but also on the choice of the regularization parameters, $\alpha$ and $\beta$. The result of the test confirms that the degree of smoothing associated with our choice of regularization parameters is very modest and does
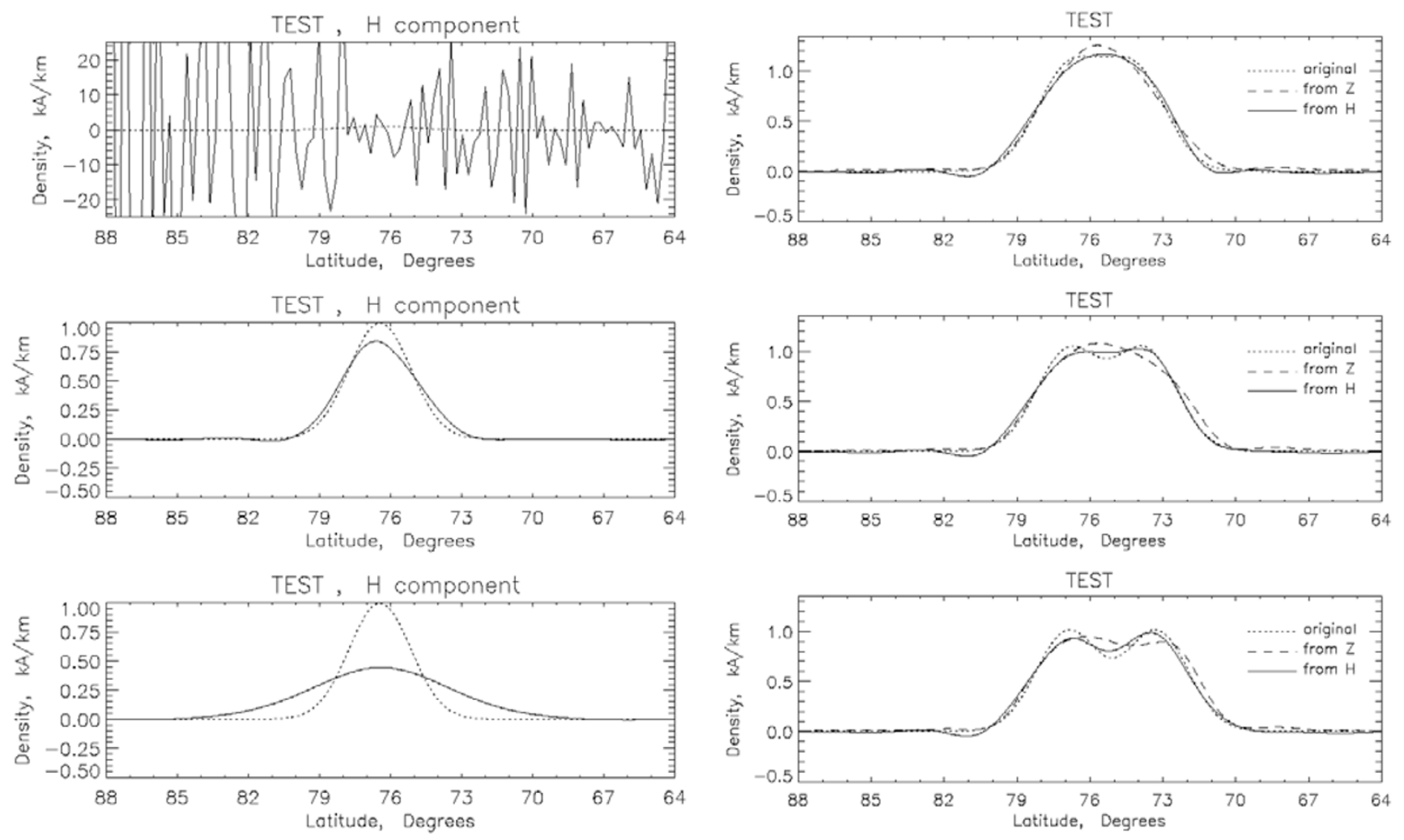

Fig. 1. Reconstruction of ionospheric currents along the meridian using Eq. (6) with different values of the regularization parameter, $\alpha=0,10^{-6}$, and 1 (from top to bottom). The original current distribution is plotted as dotted lines; note a different scale in the top panel.

Fig. 2. Reconstruction of a double-peak initial current composed of two Gaussian distributions with equal amplitudes, $j_{0}=1 \mathrm{~A} / \mathrm{m}$ and equal widths, $d_{0}=1.8^{\circ}$. The centers are separated by a distance of $1.5 \cdot 1.82^{\circ}$ (top panel), $1.75 \cdot 1.82^{\circ}$ (center), and $2.0 \cdot 1.82^{\circ}$ (bottom panel). 
Table 1. Accuracy of the reconstruction algorithm for different positions of the current center with respect to one of the two innermost stations.

\begin{tabular}{ccccccc}
\hline Reconstruction & \multicolumn{3}{c}{ from $H$} & \multicolumn{3}{c}{ from $Z$} \\
\hline$D$ & 0 & $1 / 4$ & $1 / 2$ & 0 & $1 / 4$ & $1 / 2$ \\
$d_{0}=0.9^{\circ}$ & $71 / 104$ & $64 / 107$ & $57 / 109$ & $59 / 117$ & $69 / 115$ & $73 / 110$ \\
$d_{0}=1.8^{\circ}$ & $94 / 101$ & $89 / 101$ & $86 / 101$ & $90 / 106$ & $96 / 105$ & $100 / 106$ \\
$d_{0}=3.6^{\circ}$ & $100 / 99$ & $97 / 99$ & $96 / 99$ & $102 / 106$ & $105 / 107$ & $105 / 104$ \\
$d_{0}=7.2^{\circ}$ & $100 / 95$ & $99 / 95$ & $99 / 95$ & $104 / 102$ & $105 / 102$ & $105 / 100$ \\
\hline
\end{tabular}

Note: The reconstructed "peak/total" current values are given in percents of the respective intensities of the original current (see text).

Table 2. Position of the reconstructed electrojet centers identified by the strip number (ranging from 1 to 100 , left column) for various current widths.

\begin{tabular}{ccccccccc}
\hline Reconstruction & \multicolumn{3}{c}{ from $H$} & \multicolumn{5}{c}{ from $Z$} \\
\hline$d_{0}$ & 0.9 & 1.8 & 3.6 & 7.2 & 0.9 & 1.8 & 3.6 & 7.2 \\
$\# 47$ & 47 & 47 & 47 & 47 & 48 & 48 & 49 & 50 \\
$\# 49$ & 48 & 48 & 48 & 47 & 50 & 50 & 50 & 51 \\
$\# 50-51$ & $50-51$ & $50-51$ & $50-51$ & $50-51$ & $50-51$ & $50-51$ & $50-51$ & $50-51$ \\
\hline
\end{tabular}

Note: The two innermost stations are located under the current strips \# 47 and \# 54 (see text).

not result in wiping out fine structures in the original current distribution.

Figure 3 shows results from a comparison between a simulated current of type (8) and the reconstructed electrojet for two different current center locations and widths. The solid lines represent the current distribution reconstructed from the $H$ component of the magnetic field when solving Eq. (6), and the dashed lines those from $Z$ component when solving Eq. (7).

In order to test the accuracy of the method we simulated electrojets of various widths $\left(d_{0}=0.9^{\circ}, 1.8^{\circ}, 3.6^{\circ}\right.$, and $\left.7.2^{\circ}\right)$, located at different positions with respect to the virtual stations. Table 1 shows the peak and total reconstructed currents after separation of the external and internal contributions to the magnetic field, normalized with the original peak and total current (the total current is the sum over all current strips). The center of the current distribution is initially taken to be above one of the two innermost stations, then shifted by $1 / 4$ and finally by $1 / 2$ times the distance between stations; in the latter case, the center is located exactly between the two innermost stations, i.e., the current is centered on the virtual chain. As seen from the table, the results depend on the width and position of the current. The total initial and reconstructed currents match rather well (almost always within 10\%), but the peak current densities are underestimated if the current distribution is very narrow (e.g., $0.9^{\circ}$ which is less than the spacing between virtual stations).

We performed further tests on the influence of the center of the simulation current on the reconstruction results and found that the center of the reconstructed electrojet tends to be shifted to the nearest magnetometer station when we use the $H$ component and slightly away from the nearest station when we use the $Z$ component. The results are listed in Table 2.

Figure 4 shows how a deviation from the assumption of a
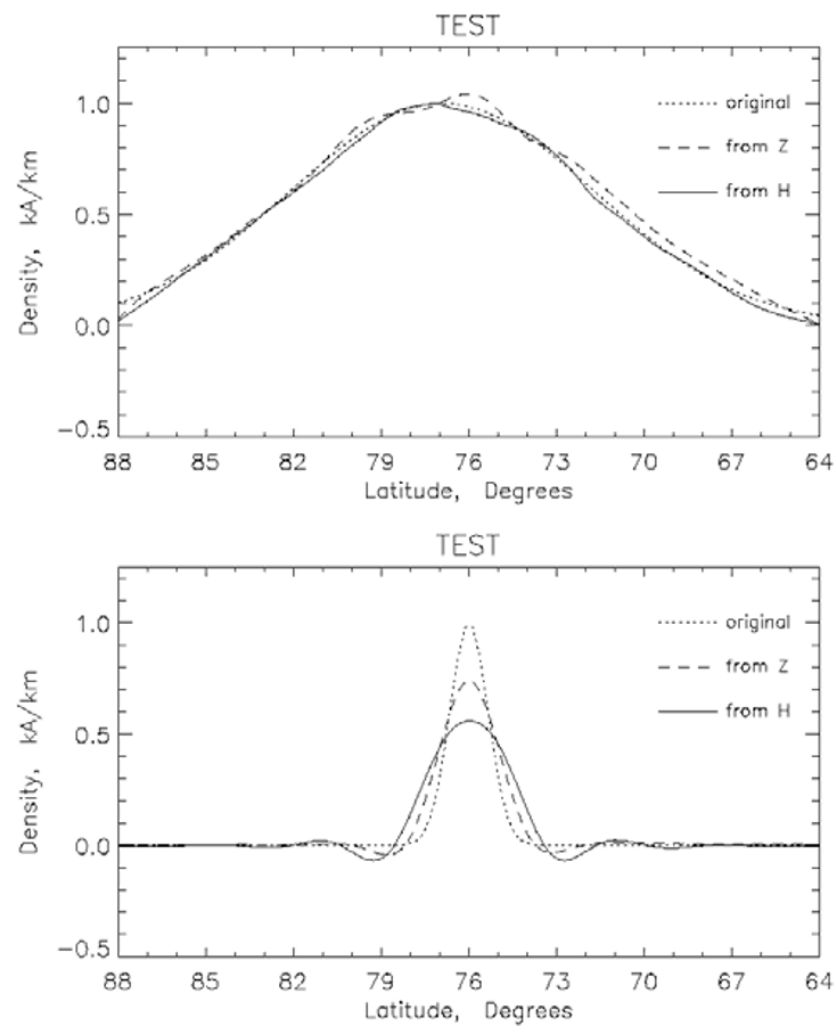

Fig. 3. Reconstruction of the ionospheric currents using Eqs. (6) and (7) with $\alpha=\beta=10^{-6}$ and different current widths. Top panel: $d_{0}=7.2^{\circ}$, $j_{0}=1 \mathrm{~A} / \mathrm{m}$, current center at $77^{\circ}$ latitude; bottom panel: $d_{0}=0.9^{\circ}$, $j_{0}=11 \mathrm{~A} / \mathrm{m}$, current center at $76^{\circ}$ latitude. The dotted curves represent the original current distribution in the form (8). The solid lines show currents reconstructed from the magnetic $H$ component and the dashed lines from the magnetic $Z$ component. 
strictly perpendicular current flow influences the result. We simulated a current distribution of the type used above, with $d_{0}=1.8^{\circ}$. A non-perpendicular current results in a non-zero eastward magnetic field component $(E)$. Here we used three different magnetic field directions for reconstruction of the current, namely northward $(H)$ and vertical $(Z)$ as before, and also total horizontal component, $F=\sqrt{H^{2}+E^{2}}$ (note that the latter component is useful only if $|E| \ll|H|$ and we preserve the same sign for both the $H$ and $F$ components). The top panel shows the reconstructed total "east-west" current, inferred from the $Z, H$, and $F$ components and normalized with the "east-west" component of the original current, as a function of the angle between original current flow and chain orientation. The bottom panel shows a comparison between peak intensities. In all cases, the total "east-west" current is overestimated and the peak "east-west" current underestimated (except for angles close to $90^{\circ}$ ). Estimation of the current from the northward magnetic field component works rather well (less than $10 \%$ error) up to $60^{\circ}$ as far as the total current is concerned, and up to $25^{\circ}$ as far as the peak current is concerned. The other components $(Z$ and $F$ ) return errors of less than $10 \%$ for angles up to $30^{\circ}$ (total current) and $40^{\circ}-50^{\circ}$ (peak current).

\section{Discussion of a Case Study}

The technique described above has been utilized in developing an interactive, on-line interface to the Greenland west coast magnetometer data covering the period from 1991 through 1999. For this study, we applied the technique to the data from 20 March 1999, a date suggested by the U. S. NOAA Space Environment Center as a candidate for the Electrojet Prediction Challenge. However, here we analyze only capabilities of the technique in monitoring the spatial distribution and dynamics of the auroral and polar cap electrojets over the Greenland west coast chain of magnetometers.

In order to get a quantitative feeling for how well the requirement for an electrojet dominated by the east-west or west-east current flow was fulfilled, we integrated for each station individually the absolute northward and eastward magnetic field variations over the entire day and then formed the ratio, $\operatorname{Int}(E) / \operatorname{Int}(H)$. The ratio varies between 0.29 (station SKT) and 1.28 (station UPN). However, the stations where the ratio is high (the northern sites) are characterized by small overall magnetic field amplitudes and consequently electrojet intensities, and the results therefore represent ratios between small numbers. Therefore it makes more sense to weight the ratio at each station by $\operatorname{Int}(H)$ from the station with the most intense magnetic field variation. In doing so, we obtain ratios between 0.29 (station SKT) and 0.49 (station UPN). Our simulation tests have shown that deviations from perpendicularity up to about $25^{\circ}$, i.e., ratios $E / H \leq 0.47$, do little harm to our method (see Fig. 4).

According to the interplanetary magnetic field (IMF) data from the NASA spacecraft ACE (ballistically propagated to the nominal magnetopause position at $\sim 12$ Earth radii), the IMF $B_{z}$ component was southward during most of the day, varying around $-5 \mathrm{nT}$ at daytime (0800-1200 UT). The $B_{z}$ component suddenly turned to zero at $1230 \mathrm{UT}$ and then varied rapidly through 2000 UT to positive values at the end
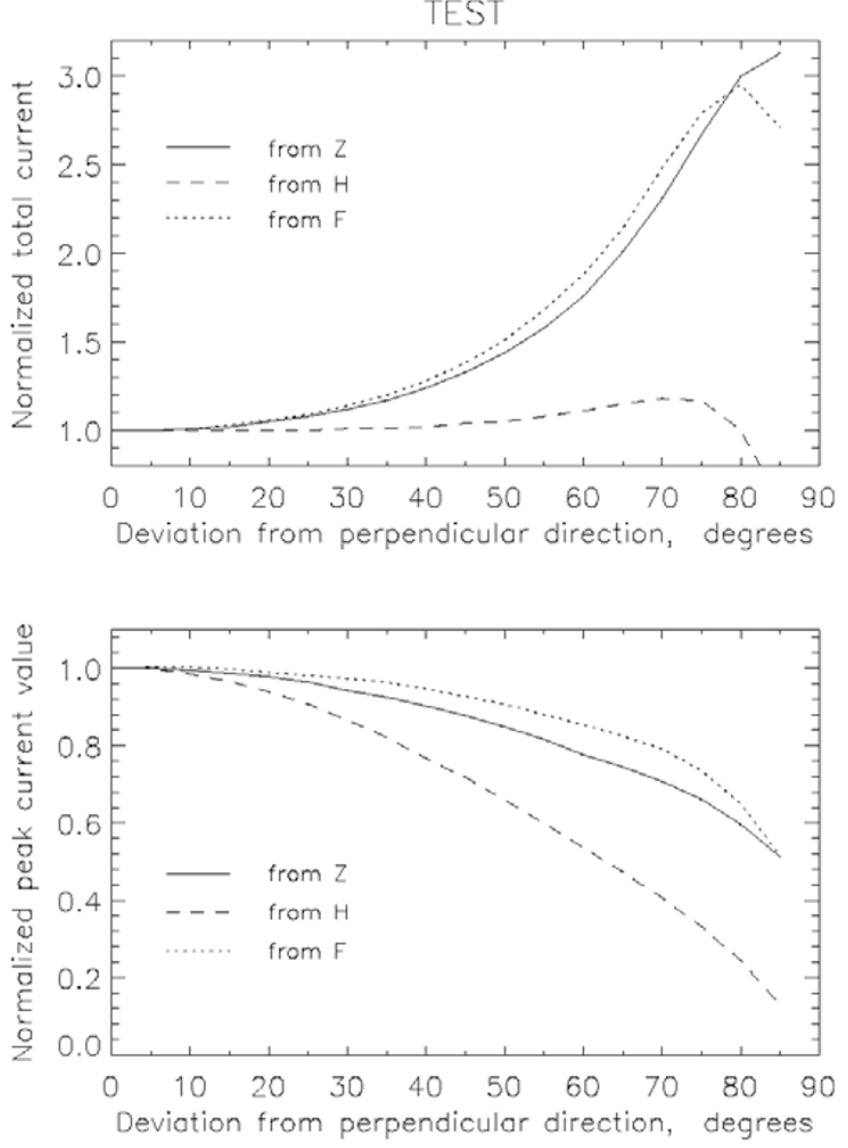

Fig. 4. Dependence of the current reconstruction on the angle between the magnetometer chain and the current orientation; zero angle means that the current is perpendicular to the chain. The top panel presents the total reconstructed current, normalized with the chain-perpendicular component of the original current. The bottom panel presents the normalized peak intensity in a similar way. Results are derived using the vertical component $Z$ (solid), the local magnetic northward horizontal component $H$ (dashed) and $F=\sqrt{H^{2}+E^{2}}$ (dotted) where $E$ denotes the geomagnetic eastward component.

of the day. The IMF $B_{y}$ component was mostly positive near $4 \mathrm{nT}$, but it changed rapidly reaching $-3 \mathrm{nT}$ at $\sim 1800 \mathrm{UT}$, then back to positive values. The solar wind velocity was low $(\sim 325 \mathrm{~km} / \mathrm{s})$ and the solar wind density varied between $2-3 \mathrm{~cm}^{-3}$, reaching 5-6 $\mathrm{cm}^{-3}$ at $1800-2000 \mathrm{UT}$.

As the Greenland magnetometer data show, a weak substorm developed over auroral latitudes at 0800 UT $(\sim 0530$ MLT at the Greenland west coast), though the major intensification in magnetic disturbances was seen at higher latitudes between 1300 and 1900 UT (magnetic noon and afternoon). At 1830 UT sharp changes occurred in the ground magnetic field over $70^{\circ}-85^{\circ} \mathrm{CGM}$ latitudes.

Figures 5, 6, and 7 show the equivalent ionospheric current densities over the Greenland west coast chain in a course of the UT day, reconstructed from 60-min averages of the magnetic northward $(H)$ component, the total horizontal field $(F)$, and the vertical $(Z)$ component, respectively. The bottom panels show the equivalent ionospheric current integrated over the chain's range (eastward - solid, westwarddotted) together with the dashed line showing the difference between the integrated currents obtained from the $H$-based 


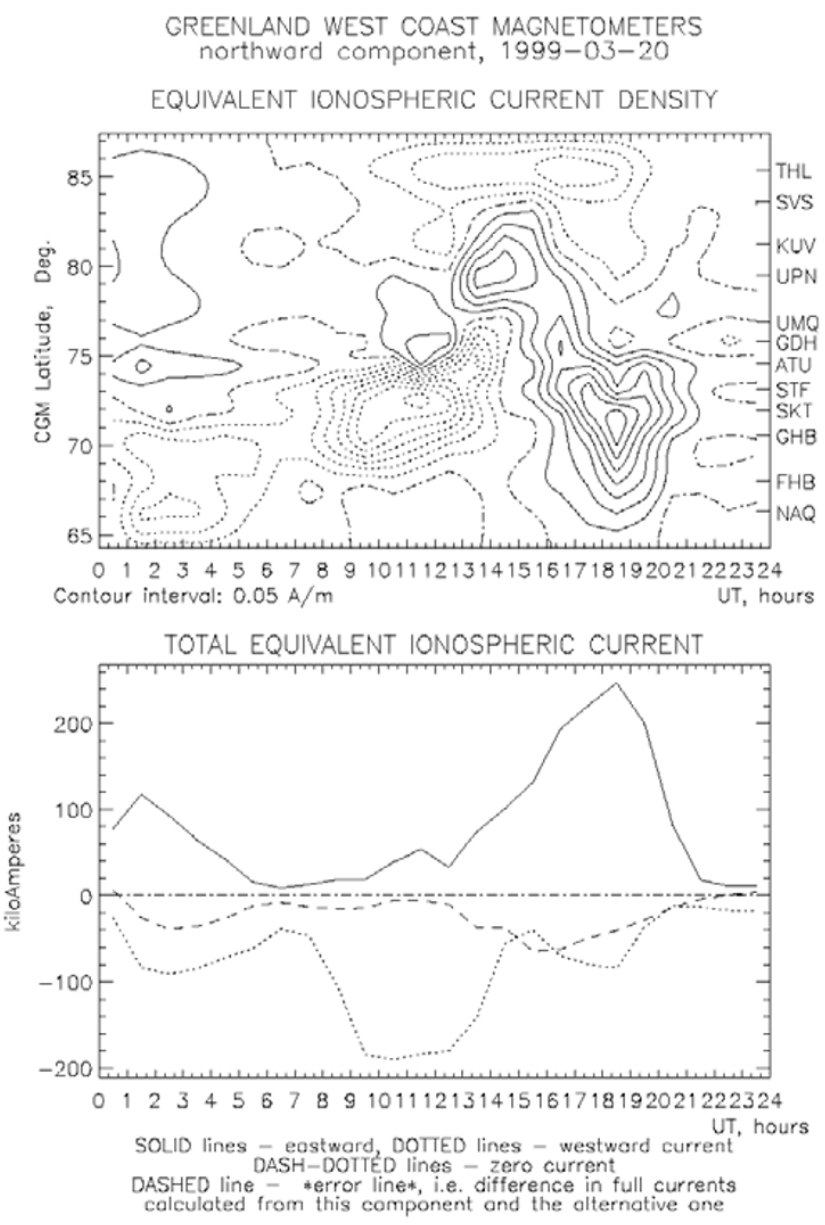

Fig. 5. Reconstructed ionospheric electrojet for 20 March 1999, obtained from the local magnetic northward $(H)$ component measured by the Greenland west coast magnetometers.

and the $Z$-based algorithms. The latter is a measure for the error of the method and is computed in the following way. First we sum the absolute values of the eastward and westward currents modeled from the $H$ (resp. $F$ ) component and the $Z$ component along the meridian, separately for each point in time. Then we subtract the latter (i.e., the $Z$-based sum) from the former (i.e., the $H$ - or $F$-based sum) for each point in time. If the difference is close to zero, the onedimensional assumption about the electrojet is a reasonable one.

All these plots show results that are similar in principle; the modeled eastward (solid contours and lines) and westward (dotted contours and lines) currents are well defined, and the error (dashed line, bottom panel) varies within about $10 \%$ of the total current. Note that the electrojet models obtained from the northward and total horizontal components are almost identical, though the model from the $Z$ component differs at times, possibly because of contributions from nonelectrojet currents. It is further seen in Figs. 5, 6, and 7 that the electrojet intensity is generally much higher south of the station ATU (where the magnetic field was indeed dominated by the northward component) than north of ATU.

The above-mentioned weak substorm around 0800 UT can be identified through the development of the westward
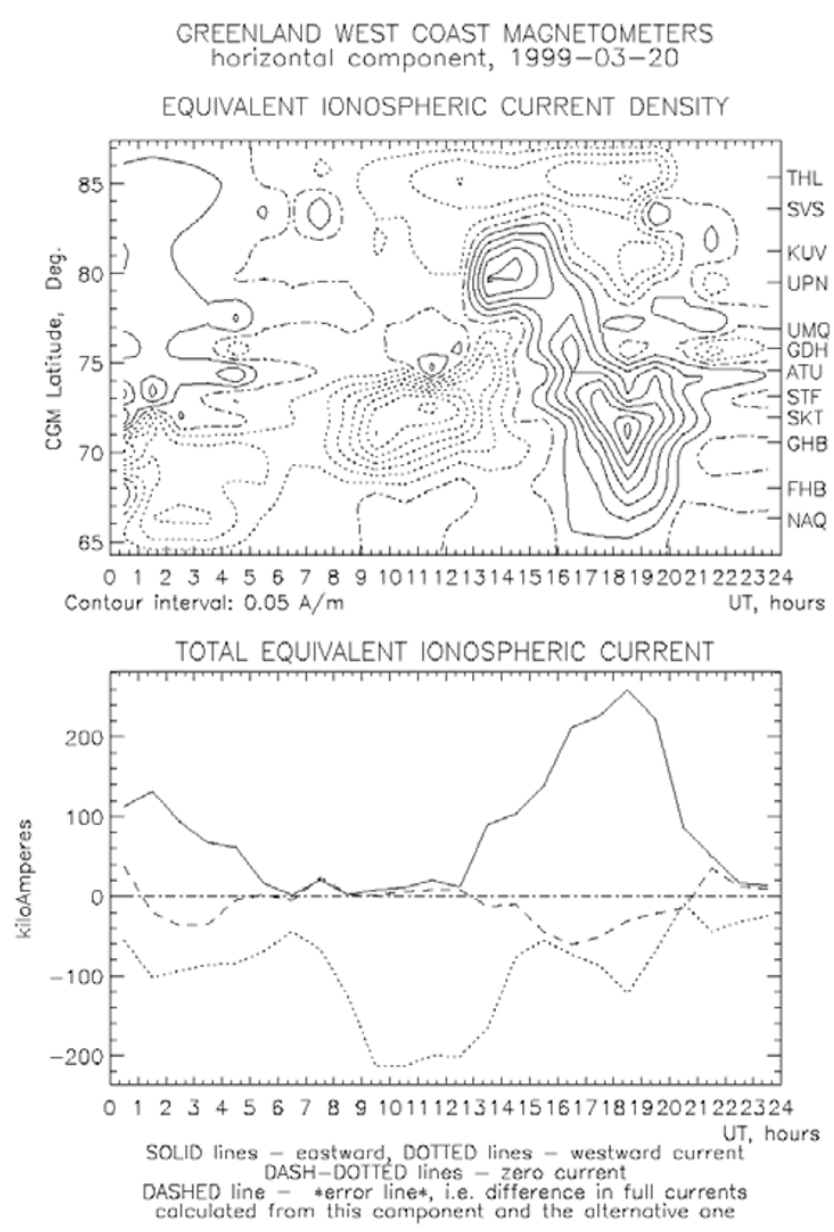

Fig. 6. As Fig. 5, but obtained from the total horizontal component $F=\sqrt{H^{2}+E^{2}}$ where $E$ is the geomagnetic eastward component.

electrojet over $68^{\circ}-75^{\circ}$ latitudes. Near local magnetic noon ( $\sim 1430 \mathrm{UT})$, the Greenland west coast chain senses the cusp electrojet (eastward according to the positive IMF $B_{y}$ component) near $80^{\circ} \mathrm{CGM}$ latitude, bounded concurrently at the equatorward side by the extensions of the westward (prenoon hours) and eastward (afternoon hours) auroral electrojets to the high latitudes. The well-defined "sandwich" structure of the cusp electrojet is seen over $77^{\circ}-85^{\circ} \mathrm{CGM}$ latitudes at noon (e.g., Rostoker, 1980; Papitashvili and Popov, 1982). Changes in the IMF components (and corresponding by in the solar wind dynamic pressure) near 1830 UT appear in the electrojet pattern as a peak developing in the eastward electrojet. We can conclude from the presented modeling results that the technique described here allows us to study and/or monitor continuously the spatial distribution and dynamics of the auroral and polar electrojets in detail, changing interactively the data averaging and contour intervals.

\section{Conclusions}

In this study, we investigated a technique to model the "east-west" equivalent ionospheric current from a meridianaligned magnetometer chain. Our results from the simulation studies can be summarized as follows. 

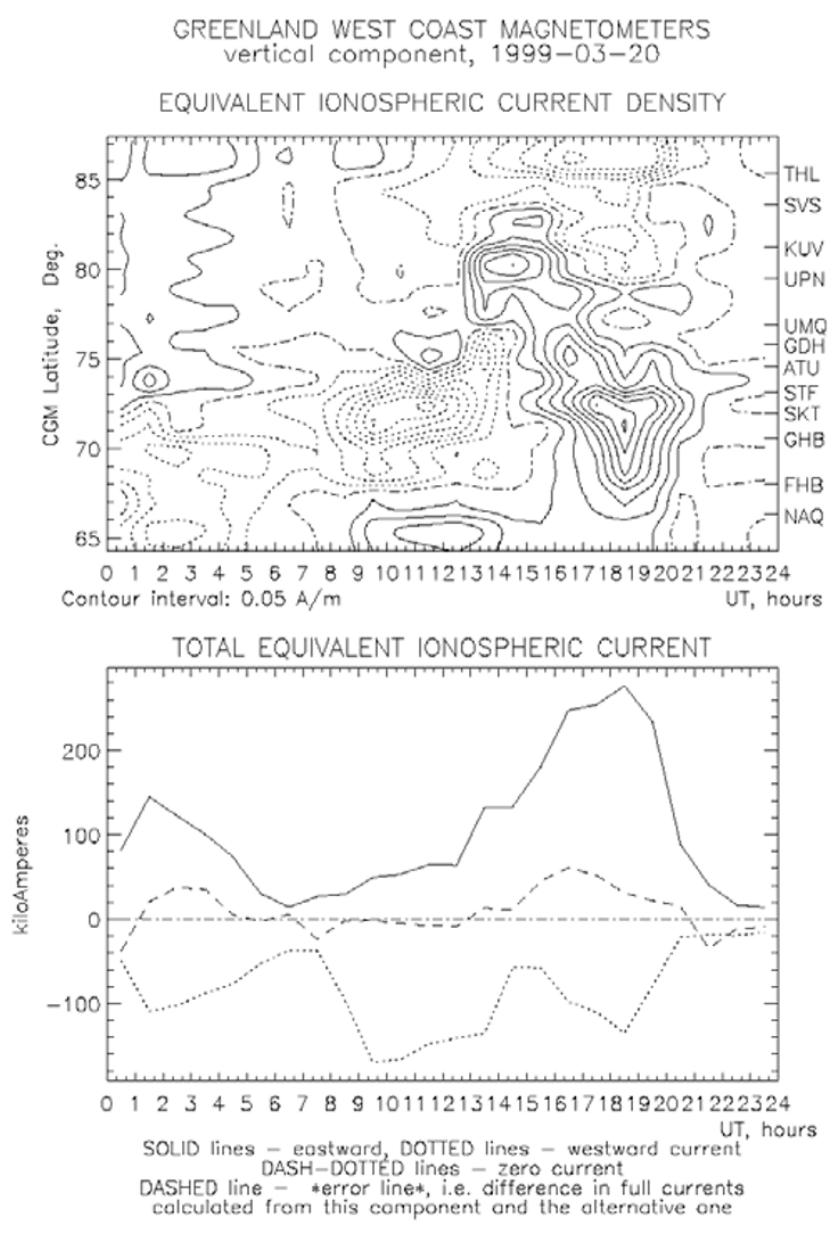

Fig. 7. As Fig. 5, but obtained from the vertical $(Z)$ geomagnetic field component.

1) The technique slightly underestimates the true peak current density if we use solely the magnetic north component for reconstructing the spatial distribution of the current; we slightly overestimate the peak current if we use solely the vertical component.

2) The method allows resolving the spatial distribution of the current density with good accuracy when the current is fully confined within the chain range and the width of the current is more than the distance between stations. For narrower distributions (less than the distance between stations), the algorithm produces too small peak amplitudes and too wide distributions.

3) The method reconstructs well a double-peaked electrojet when the peak separation is more than twice the distance between adjacent stations. For smaller spatial structures (less than twice the distance between stations), the algorithm produces smaller peak amplitudes and wider distributions than were originally used.

4) If the electrojet flows under a certain angle with respect to the meridian (that is, not exactly perpendicular to the magnetometer chain), the total "east-west" current reconstructed from the $H$ component is practically independent of the true current direction for angles up to $60^{\circ}$. The peak values are underestimated consistently and increasingly; the total currents are overestimated if the angle increases from $0^{\circ}$ (a current flows perpendicular to the chain) to close to $90^{\circ}$ (a current flows along the chain). The error is small for angles less than some $30^{\circ}$. This applies to reconstruction from any component, $H$, $Z$, or $F$.

The results of this study show that the proposed method yields a good model of the equivalent ionospheric current flowing across the meridian chain of magnetometers. The current density contour plots over the range of CGM latitudes versus UT hours can serve as a powerful tool for monitoring these currents when studying the dynamics of the auroral and polar (cusp) electrojets during magnetic storms and substorms.

In order to gain more experience with electrojet modeling and be able to better assess the validity of our method, we intend to collaborate with the Scandinavian IMAGE magnetometer team on comparing two different 1-D electrojet estimation methods, the upward field continuation used by Mersmann et al. (1979) and the algorithm presented in this paper. We plan to apply both techniques to the same observed data samples and expect to obtain quantitative results on the relative performance of the methods.

Acknowledgments. We thank the U.S. NOAA Space Environment Center for providing near-real time IMF data from NASA's ACE spacecraft on-line. This work has been performed with support from the NSF awards OPP-9614175, ATM-9727554, and OPP-9876473 to the University of Michigan and the Ørsted satellite project funded by the Danish Government. The Editor thanks O. Amm and D. H. Boteler for their assistance in evaluating this paper.

\section{References}

Amm, O., Ionospheric elementary current systems in spherical coordinates and their application, J. Geomag. Geoelectr., 49, 947-955, 1997.

Amm, O. and A. Viljanen, Ionospheric disturbance magnetic field continuation from the ground to the ionosphere using spherical elementary current systems, Earth Planets Space, 51, 431-440, 1999.

Baumjohann, W., R. J. Pellinen, H. J. Opgenoorth, and E. Nielsen, Joint twodimensional observations of ground magnetic and ionospheric electric fields associated with auroral zone currents: current systems associated with the local break-ups, Planet. Space Sci., 29, 431-447, 1981.

Boteler, D. H. and R. J. Pirjola, The complex image method for calculating the magnetic and electric fields produced at the surface of the Earth by the auroral electrojet, Geophys. J. Int., 132, 31-40, 1998.

Engels, M., Untersuchungen zur elektromagnetischen Induktion in Grönland, 131 pp., Ph.D. Thesis, Georg-August-Universität Göttingen, Cuvillier Verlag, Göttingen, 1997 (in German).

Gustafsson, G., N. E. Papitashvili, and V. O. Papitashvili, A revised corrected geomagnetic coordinate system for Epochs 1985 and 1990, J. Atmos. Terr. Phys., 54, 1609-1631, 1992.

Kamide, Y., A. D. Richmond, and S. Matsushita, Estimation of ionospheric electric fields, ionospheric currents and field-aligned currents from ground magnetic records, J. Geophys. Res., 86, 801-813, 1981.

Kisabeth, J. L. and G. Rostoker, Development of the polar electrojet during polar magnetic substorms, J. Geophys. Res., 76, 6815-6828, 1971.

Korobeinikov, V. G., V. G. Petrov, and V. O. Papitashvili, Algorithm for derivation of polar electrojet parameters by the use of meridional chain data, Geomagnetic Research, 21, 41-45, Sovetskoe Radio, Moscow, 1977 (in Russian).

Kotikov, A. L., Yu. A. Latov, and O. A. Troshichev, Structure of auroral electrojets by the data from a meridional chain of magnetic stations, Geophysica, 23, 143-154, 1987.

Kotikov, A. L., B. D. Bolotynskaya, V. A. Gizler, O. A. Troshichev, A. B. Pashin, and V. R. Tagirov, Structure of auroral zone phenomena from 
the data of meridional chains of stations: magnetic disturbances in the nighttime auroral zone and auroras, J. Atmos. Terr. Phys., 53, 265-277, 1991.

Kuznetsov, B. M., O. A. Troshichev, and V. A. Zhilenkov, A study of the auroral electrojets structure, Geomagnetic Research, 23, 91-98, Sovetskoe Radio, Moscow, 1978 (in Russian).

Mareschal, M., On the problem of simulating the Earth's induction effects in modeling polar magnetic substorms, Rev. Geophys. Space Phys., 14, 403-410, 1976.

Mersmann, U., W. Baumjohann, F. Kuppers, and K. Lange, Analysis of an eastward electrojet by means of upward continuation of ground-based magnetometer data, J. Geophys., 45, 281-298, 1979.

Olsen, N., A new tool for determining ionospheric currents from magnetic satellite data, Geophys. Res. Lett., 23, 3635-3638, 1996.

Papitashvili, V. O. and V. A. Popov, Ionospheric currents in the southern polar cusp as a function of the sign of the azimuthal component of the IMF, Geomagn. Aeron., 22, Engl. Transl., 264-265, 1982.

Popov, V. A. and Ya. I. Feldstein, On a new interpretation of "Harang discontinuity", Geomagn. Aeron., 36, 43-51, 1996 (in Russian).

Pudovkin, M. I., Sources of bay-like disturbances, Izv. Acad. Sci., Ser. Geophys., 3, (Trans. Acad. Sci. USSR, Geophysics), 484-489, 1960 (in Russian).
Richmond, A. D. and W. Baumjohann, Three-dimensional analysis of magnetometer array data, J. Geophys., 54, 138-156, 1983.

Rostoker, G., Magnetospheric and ionospheric currents in the polar cusp and their dependence on the By component of the interplanetary magnetic field, J. Geophys. Res., 85, 4167-4176, 1980.

Tikhonov, A. N. and V. Ia. Arsenin, Solution of Ill-Posed Problems, 258 pp., Halsted Press, New York, 1977.

Untiedt, J. and W. Baumjohann, Studies of polar current systems using the IMS Scandinavian magnetometer array, Space Sci. Rev., 63, 245-390, 1993.

Viljanen, A., O. Amm, and R. Pirjola, Modeling geomagnetically induced currents during different ionospheric conditions, J. Geophys. Res., 104, 28,059-28,071, 1999.

Walker, J. K., V. Semenov, and T. L. Hansen, Synoptic models of high latitude magnetic activity and equivalent ionospheric and induced currents, J. Atmos. Terr. Phys., 59, 1435-1452, 1997.

Weaver, J. T., On the separation of local geomagnetic fields into external and internal parts, Z. Geophys., 30, 29-36, 1964.

V. A. Popov (e-mail: vpo@dmi.dk), V. O. Papitashvili (e-mail: vp@dmi. dk), and J. F. Watermann (e-mail: jfw@dmi.dk) 